\title{
MS03-P16 | Dynamic Formation And Internal Order Of Liquid Dense Protein Clusters Beyond Crystallography
}

Falke, Sven (University Hamburg, Hamburg, GER); Brognaro, Hevila ; Mudogo, Celestin Nzanzu ; Wang, Mengying ; Cheng, Qingdi ; Betzel, Christian (University Hamburg, Institute for Biochemistry and Molecular Biology, Hamburg, GER)

Ellipsoidal liquid-dense clusters (LDCS) can be formed by liquid-liquid phase separation, which is a process known in vitro as initial step in crystal nucleation [1]. A rapidly increasing number of studies also describe dense protein or nucleic acid clusters in vivo, mainly formed in the context of RNA signaling, stress, neurodegenerative diseases like Alzheimer and potentially even in vivo crystallization [1]. However, insights into structure and dynamics of macromolecules inside LDCs and how to induce or manipulate molecular order are till now missing. Insights will not only provide new options to optimize protein crystal growth but will also potentially provide new routes to utilize LDCs of nanomaterials for structural investigation in a non-crystalline environment in the future, for example applying particle imaging methods at highly brilliant radiation sources, including coherent X-ray diffractive imaging (CXD).

In order to investigate formation and internal structure of LDCs we apply particularly DLS, EM, CD spectroscopy, particle tracking and X-ray imaging techniques. We selected proteins of medical and biological relevance, including a $\beta$-lactamase, concanavalin A, $\beta$-lactoglobulin, TbIMPDH and tau, as well as RNAs to prepare and analyse LDCs. The physico-chemical conditions for formation, stabilization and labelling of selected macromolecule LDCs were systematically established in multi-dimensional phase diagrams. Further, external electromagnetic fields have been applied to analyse the response towards two-dimensional ordering. Further details will be presented.

[1] Falke, S. et al. (2018). Encyclopedia of Analytical Chemistry, Vol. edited by R.A. Meyers, pp. 1-25. Chichester, UK: John Wiley \& Sons, Ltd. 